\title{
Sequencing of Sclerosing Microcystic Adenocarcinoma Identifies Mutational Burden and Somatic Variants Associated With Tumorigenesis
}

\author{
ROY JIANG ${ }^{1}$, JONATHAN MARQUEZ ${ }^{2}$, JACOB I. TOWER ${ }^{3}$, DANIEL JACOBS ${ }^{3}$, \\ WENQIAN CHEN ${ }^{4}$, SARAL MEHRA ${ }^{3}$, MANJU LATA PRASAD ${ }^{4}$ and BENJAMIN L. JUDSON ${ }^{3}$ \\ ${ }^{1}$ Department of Immunobiology, Yale School of Medicine, New Haven, CT, U.S.A.; \\ ${ }^{2}$ Department of Genetics, Yale School of Medicine, New Haven, CT, U.S.A.; \\ ${ }^{3}$ Division of Otolaryngology, Department of Surgery, Yale School of Medicine, New Haven, CT, U.S.A.; \\ ${ }^{4}$ Department of Pathology, Yale School of Medicine, New Haven, CT, U.S.A.
}

\begin{abstract}
Background/Aim: Sclerosing microcystic adenocarcinoma (SMA) is a rare oral cavity neoplasia, histologically resembling microcystic adnexal carcinoma (MAC) of the skin. Only nine SMA cases have been reported in the literature, frequently in the context of immunosuppression; SMA has not been recognized in the most recent WHO tumor classification. We sought to identify potential molecular mechanisms of tumorigenesis in a case of SMA relative to those known for MAC. Case Report: A 41-year-old female with psoriatic arthritis undergoing immunosuppression therapy presented with a tongue mass. Biopsy revealed a diagnosis of SMA. Partial glossectomy and neck dissection showed no residual tumor or nodal disease. Results: whole exome sequencing revealed moderate mutational burden and putative loss of function mutations in CDK11B but no overlap with known MAC mutations. Conclusion: We characterized the genomic profile of SMA for the first time, identifying both mutational burden and unique somatic variants associated with tumorigenesis.
\end{abstract}

Sclerosing microcystic adenocarcinoma (SMA) is a rare neoplasm that histologically resembles microcystic adnexal carcinoma (MAC) but is found in extracutaneous areas of the head and neck without adnexal associations (1-3). Like MAC, SMA is characterized by thin nests and cords of

This article is freely accessible online.

Correspondence to: Saral Mehra, Yale University School of Medicine, 47 College Street, Suite 216 New Haven, CT 06510, U.S.A. E-mail: saral.mehra@yale.edu

Key Words: Next-generation sequencing, sclerosing microcystic adenocarcinoma, immunosuppression, oral cancer. squamous/basaloid cells including ducts and cystic spaces suggesting a possible origin from eccrine sweat glands or the pilosebaceous-apocrine unit $(4,5)$. Both are also characterized by local infiltration with perineural invasion.

SMA was first established as an entity distinct from MAC in a five-patient case series, including one case in the context of immunosuppression (acute myeloid leukemia or AML treatment) (1). A separate case of SMA was identified in the context of a patient diagnosed with multiple sclerosis previously treated with the immunosuppressive agent, glatiramer (6). Current SMA diagnosis relies heavily on histological evaluation and no studies have yet attempted the molecular characterization of this entity. In this study, we characterized the molecular characteristics of SMA in a case identified in the context of a patient undergoing therapeutic immunosuppression for autoimmunity.

\section{Case Report}

A 41-year-old female presented to her primary care physician with a chief complaint of a "bump inside her tongue". She noticed a right tongue tip lesion 1 week before and also reported her right lip to be swollen. The patient was a former smoker of 20 years. The patient was also being treated with apremilast (PDE4 inhibitor) for her psoriatic arthritis and had previously been treated with methotrexate, infliximab and adalimumab.

She was subsequently referred to an otolaryngologist for a biopsy. The lesion was described as a 3-5 mm palpable firm nodule that was relatively intact with overlying mucosa. The lesion had no effect on chewing and swallowing and was not painful with no drainage. An excisional biopsy was performed. The sublingual specimen was $9 \mathrm{~mm}$ in size. Histologically the lesion showed an infiltrative growth pattern with desmoplastic stroma and extensive perineural invasion; tubules, cords and strands of tumor cells infiltrating muscle, nerves and minor 

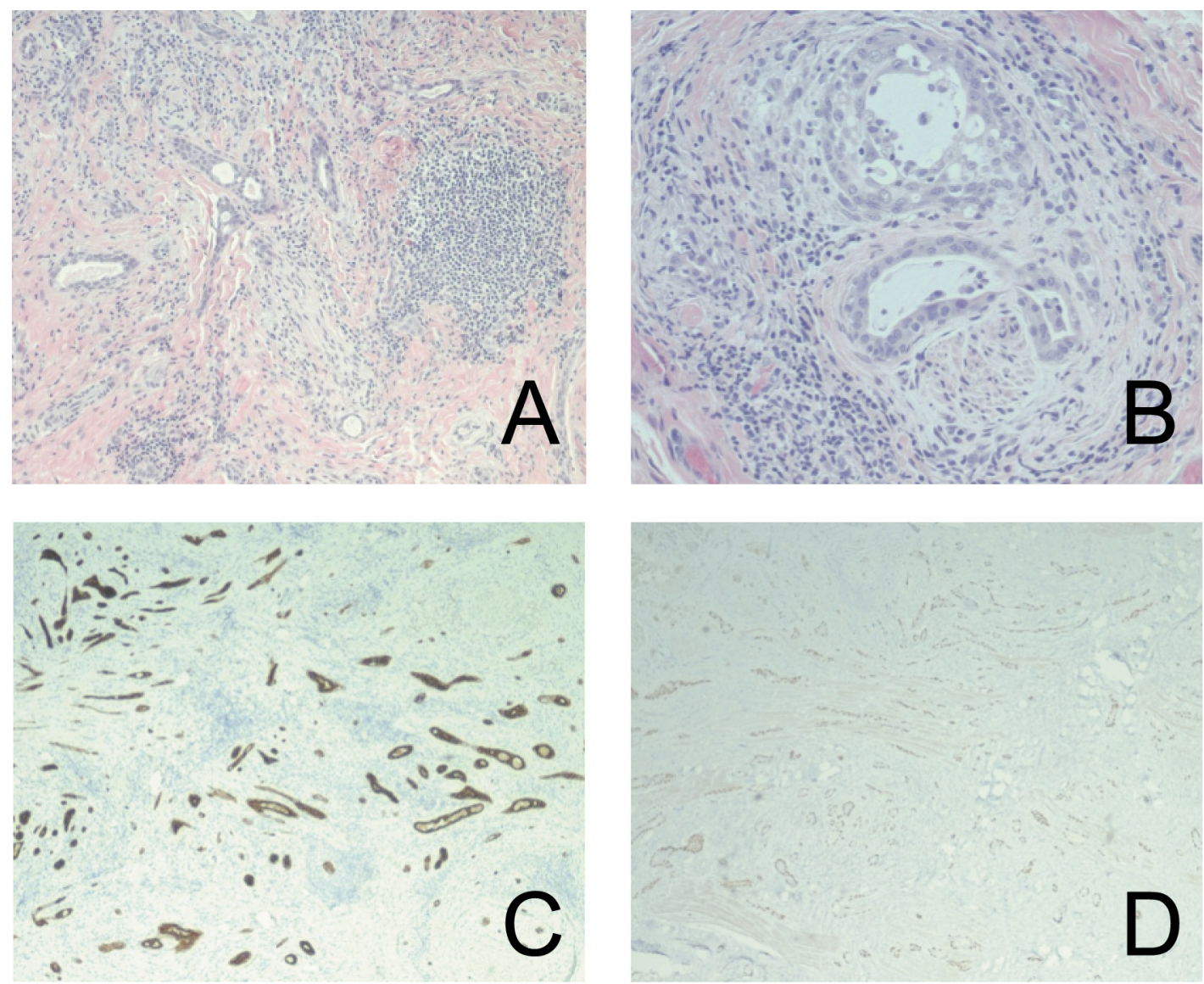

Figure 1. Histopathological characterization of SMA sample. (A) H\&E stain shows infiltrative tumor cells forming tubules; (B) H\&E staining shows perineural invasion; (C) Immunohistochemistry for CK7 is strongly diffusely positive; (D) Immunohistochemistry for p63 highlights basal cell layer.

salivary glands were observed (Figure 1A, B and C). Tubules were angulated and lined by two layers of bland cells. The luminal cuboidal epithelial cells were CK7+ and abluminal basal cells were both p40+ p63+ (Figure 1D). Tubules displayed a microcystic pattern but mitosis and necrosis associated with high grade malignancy was not identified. The differential diagnosis included polymorphous adenocarcinoma, adenoid cystic carcinoma and sclerosing mucoepidermoid carcinoma. However, the simultaneous expression of p63 and p40 was inconsistent with polymorphous adenocarcinoma, the absence of SOX10 and c-Kit staining was inconsistent with adenoid cystic carcinoma, and the absence of intracytoplasmic mucin and expression of p63 only by basal cells was not consistent with mucoepidermoid carcinoma. No expression of S100, mammaglobin and GATA3 was seen.

The lesion's resemblance to MAC and mucosal location suggested a diagnosis of sclerosing microcystic adenocarcinoma (SMA). Subsequent partial glossectomy of the scar and tumor bed revealed only post-surgical fibrosis and giant cell reaction but no residual tumor. Bilateral neck dissection of levels 1-3 revealed no nodal disease or submandibular gland involvement. Subsequent PET/CT was negative for abnormal hypermetabolism of the anterior tongue with only small mildly avid hypermetabolic cervical lymph nodes.

To characterize genomic variation that may contribute to SMA tumorigenesis, we performed whole exome sequencing (WES) of SMA tumor tissue and paired blood from the patient. Importantly, we had no reason to believe that tumorigenesis was mediated via germline mutations as no extraglossal lesions were evident and no family history of cancer was recorded. We assessed for rare somatic variants present only in tumor tissue but absent in control tissue. A total of 124 unique missense mutations were identified. Based on our analysis of these mutations, we report a set of somatic mutations: (A) 17 that likely lead to gene dysfunction based on the intrinsic nature of the change resulting from the mutation (Table I) and (B) 14 that likely lead to gene dysfunction based on predicted consequences of coding missense mutations consistent (Table II). Interestingly, the candidate mutations were all novel variants not previously described in the literature. However, a 
Table I. Table of putative loss of function mutations in SMA sample.

\begin{tabular}{|c|c|c|c|c|c|c|c|c|c|}
\hline Location & Variant & Consequence & Symbol & Type & Exon & Intron & $\begin{array}{l}\text { Coding } \\
\text { sequence } \\
(\mathrm{CDS})\end{array}$ & Protein & $\begin{array}{c}\text { AA } \\
\text { Change }\end{array}$ \\
\hline 1:1580837-1580837 & A & Stop gained & CDK11B & Protein coding & $6 / 21$ & - & 445 & 149 & $\mathrm{Q} / *$ \\
\hline 1:11898953-11898953 & $\mathrm{C}$ & Frameshift & CLCN6 & Protein coding & $22 / 22$ & - & $2765-2766$ & 922 & S/SX \\
\hline 1:16912095-16912095 & A & Stop gained & NBPF1 & Protein coding & $12 / 29$ & - & 877 & 293 & $\mathrm{Q} / *$ \\
\hline 1:144825789-144825789 & $\mathrm{C}$ & Splice donor & NBPF9 & Protein coding & - & $11 / 12$ & - & - & - \\
\hline 1:231990628-231990628 & $\mathrm{T}$ & Frameshift & DISC1 & Protein coding & $10 / 10$ & - & $2125-2126$ & 709 & N/IX \\
\hline $2: 219250210-219250210$ & $\mathrm{~T}$ & Splice donor & SLC11A1 & NMD & - & $5 / 15$ & - & - & - \\
\hline 11:65819075-65819075 & G & Splice donor & SF3B2 & Processed transcript & - & $2 / 2$ & - & - & - \\
\hline 12:7917985-7917985 & $\mathrm{G}$ & Splice donor & NANOGNB & Protein coding & - & $1 / 3$ & - & - & - \\
\hline $15: 28950628-28950628$ & $\mathrm{~T}$ & Splice donor & GOLGA8M & Protein coding & - & $12 / 18$ & - & - & - \\
\hline $16: 28769317-28769317$ & $\mathrm{~T}$ & Splice acceptor & NPIPB9 & Protein coding & - & $1 / 7$ & - & - & - \\
\hline 17:5118133-5118133 & $\mathrm{C}$ & Splice acceptor & SCIMP & Protein coding & - & $4 / 4$ & - & - & - \\
\hline 19:20990109-20990109 & G & Frameshift & ZNF66 & Protein coding & $4 / 4$ & - & $1703-1704$ & 568 & $\mathrm{E} / \mathrm{EX}$ \\
\hline 19:39883291-39883291 & A & Splice acceptor & MED29 & NMD & - & $2 / 3$ & - & - & - \\
\hline $21: 15311565-15311565$ & $\mathrm{~T}$ & Splice donor & ANKRD20A11P & Transcribed pseudogene & - & $14 / 24$ & - & - & - \\
\hline $22: 23498331-23498331$ & AA & Frameshift & RAB36 & Protein coding & $4 / 4$ & - & $389-390$ & 130 & $\mathrm{~K} / \mathrm{KX}$ \\
\hline$X: 44336206-44336206$ & A & Splice donor & FDPSP5 & Transcribed pseudogene & - & $1 / 1$ & - & - & - \\
\hline$X: 135960145-135960145$ & AA & Frameshift & RBMX & Protein coding & $4 / 9$ & - & $316-317$ & 106 & P/LX \\
\hline
\end{tabular}

NMD: Nonsense-mediated decay; AA: amino acid.

Table II. Table of missense mutations in SMA sample.

\begin{tabular}{|c|c|c|c|c|c|c|c|c|}
\hline Location & Variant & Consequence & Symbol & Type & Exon & $\begin{array}{l}\text { Coding } \\
\text { sequence } \\
(\mathrm{CDS})\end{array}$ & Protein & $\begin{array}{c}\text { AA } \\
\text { Change }\end{array}$ \\
\hline 1:47610292-47610292 & $\mathrm{T}$ & Missense & CYP4A22 & Protein coding & $8 / 12$ & 968 & 323 & $\mathrm{P} / \mathrm{L}$ \\
\hline 1:145299757-145299757 & $\mathrm{T}$ & Missense & NBPF10 & NMD & $6 / 47$ & 806 & 269 & $\mathrm{~T} / \mathrm{I}$ \\
\hline 1:146057426-146057426 & A & Missense & NBPF11 & Protein coding & $6 / 26$ & 24 & 8 & $\mathrm{~W} / \mathrm{C}$ \\
\hline 2:114257161-114257161 & $\mathrm{C}$ & Missense & FOXD4L1 & Protein coding & $1 / 1$ & 328 & 110 & $\mathrm{~N} / \mathrm{H}$ \\
\hline 6:136590612-136590612 & $\mathrm{C}$ & Missense & BCLAF1 & Protein coding & $9 / 12$ & 2176 & 726 & $\mathrm{~K} / \mathrm{E}$ \\
\hline $7: 76241067-76241067$ & $\mathrm{~T}$ & Missense & POMZP3 & Protein coding & $2 / 4$ & 184 & 62 & $\mathrm{~A} / \mathrm{T}$ \\
\hline $9: 119802148-119802148$ & $\mathrm{~A}$ & Missense & ASTN2 & Protein coding & $5 / 22$ & 1220 & 407 & $\mathrm{P} / \mathrm{L}$ \\
\hline $10: 29783855-29783855$ & $\mathrm{~T}$ & Missense & SVIL & Protein coding & $22 / 40$ & 3829 & 1277 & $\mathrm{~V} / \mathrm{M}$ \\
\hline $11: 71806842-71806842$ & $\mathrm{~T}$ & Missense & LRTOMT & Protein coding & $2 / 2$ & 137 & 46 & $\mathrm{P} / \mathrm{L}$ \\
\hline $16: 21848438-21848438$ & $\mathrm{G}$ & Missense & NPIPB4 & Protein coding & $1 / 2$ & 622 & 208 & $\mathrm{D} / \mathrm{H}$ \\
\hline $17: 45234297-45234297$ & $\mathrm{C}$ & Missense & $\mathrm{CDC} 27$ & NMD & $7 / 12$ & 824 & 275 & $\mathrm{P} / \mathrm{R}$ \\
\hline $18: 47363929-47363929$ & $\mathrm{G}$ & Missense & MYO5B & Protein coding & $37 / 40$ & 5096 & 1699 & $\mathrm{~L} / \mathrm{S}$ \\
\hline $19: 56758151-56758151$ & $\mathrm{G}$ & Missense & ZSCAN5D & Protein coding & $5 / 5$ & 779 & 260 & $\mathrm{~A} / \mathrm{G}$ \\
\hline X:118603772-118603772 & $\mathrm{C}$ & Missense & SLC25A5 & Protein coding & $2 / 4$ & 260 & 87 & $\mathrm{~L} / \mathrm{P}$ \\
\hline
\end{tabular}

NMD: Nonsense-mediated decay; AA: amino acid.

number of genes in our analysis have also previously been found to be mutated in malignancies.

To further evaluate associations between genes with somatic mutations identified in this case and other malignancies, we analyzed data from the cancer genome atlas (TCGA). The TCGA includes over 20,000 molecularly profiled primary tumor and matched normal tissue samples spanning 33 cancer types (7). We sought to compare SMA mutations that we had identified with those previously observed in other cancers. Of the genes with somatic variants uncovered in this study, CDK11B, NBPF1, SF3B2, MED29, and RAB36, have previously been associated with favorable cancer prognosis; that is, their loss of function had been correlated with worse survival. Thus, genes affected by somatic variants in this case of SMA correspond to those previously associated with poor cancer outcomes and tumorigenesis. 


\section{Discussion}

To our knowledge, this is the first study to profile SMA at the molecular level $(1,6)$. Previous studies have attempted molecular characterization of MAC; SMA has often been described as a variant of MAC owing to its histological resemblance to sweat glands and infiltrative pattern and tendency toward perineural invasion but with a more favorable prognosis $(1-3,8)$. Mutations have been identified in CDKN4/6 and also in TP53 and JAK1 genes in approximately $20 \%$ of patients with $\operatorname{MAC}(9,10)$. However, no genes previously shown to be mutated in MAC were mutated in this case report on $\operatorname{SMA}(9,10)$. Future studies need to establish a consistent lack of overlap in the mutational profile of MAC compared to SMA.

CDK family gene mutations have been observed in MAC and mutations in this large gene family were also observed here (10). CDK mutations have been widely observed in the context of tumorigenesis and CDK11 overexpression has been found in the context of breast cancer, and multiple myeloma (11). In our analysis of head and neck squamous cell carcinoma data from TCGA, elevated expression of CDK11 was associated with better overall survival. The CDK11B mutation observed in our study involved a loss of function mutation at positions 445 in its canonical coding sequence and would likely abrogate its protein kinase function.

Several previous studies have observed SMA in the context of treatment with immunosuppression. One case report involved a patient treated with glatiramer for multiple sclerosis, while another involved a patient undergoing stem cell transplantation for AML $(1,6)$. The patient in this case report previously received treatment with methotrexate, infliximab, adalimumab and apremilast. Immunosuppression is a well-established risk factor for neoplasia because of its potential effect on immune surveillance (12). To further investigate this, we also quantified the tumor mutational burden (TMB) and observed 124 unique missense somatic mutations. A cutoff of 199 missense mutations has previously been defined to correlate with the FDA-approved FoundationOne $\mathrm{Cdx}$ assay value of 10 mutations/Mb; values above 10 mutations/Mb are described as "hypermutator" status (13). TMB is a known biomarker for therapy responses in immune checkpoint blockade by promoting antitumor immune surveillance (14). It is worth speculating that the moderate TMB observed in the context of this cancer associated with immunosuppression may suggest a causative association with neoplasia. In general, high TMB manifests as cytologic changes e.g. nuclear atypia and pleomorphism which were absent in this case; these results are intriguing and inconsistent with the banal morphology.

Strengths of this investigation include the use of unbiased whole exome sequencing for identifying somatic variants associated with tumorigenesis. However, the limited generalizability of this study in examining only one case of SMA should be noted as a limitation. Given the exceptionally rare occurrence of SMA, accruing a large cohort for investigation is challenging. We hope this study motivates awareness of this entity and the future molecular profiling of larger cohorts of SMA cases through collaborative efforts.

\section{Conclusion}

Our study is the first to characterize the genomic profile of SMA, an extremely rare cancer of the oral mucosa frequently associated with immunosuppression and resembling MAC, a known entity. We sought to identify possible molecular causes of tumorigenesis and test if mutations in genes known to be mutated in MAC could also be found. We observed moderate TMB frequency and mutations associated with tumorigenesis but none shared with MAC. These findings suggest that SMA may be a distinct entity and that immunosuppression may contribute to tumorigenesis. This study also motivates the molecular profiling of SMA in the context of larger cohorts to better understand the role of immunosuppression and failed immune surveillance in causing head and neck neoplasia.

\section{Conflicts of Interest}

The Authors have no financial disclosures/conflicts of interest with regard to this study.

\section{Authors' Contributions}

Conceptualization: RJ, JM, JT, MP, BJ were responsible for the design of this study; Methodology: RJ, JM, WC, DJ performed the experiments; Formal analysis and investigation: RJ, JM, WC conducted the analysis; Writing - original draft preparation: RJ, JM, MP wrote the manuscript; Writing - review and editing: RJ, JM, DJ, JT, MP, SM, WC, BJ revised the manuscript; Funding acquisition: BJ provided funding; Resources: BJ provided resources for the study; Supervision: BJ and MP supervised the study.

\section{References}

1 Mills AM, Policarpio-Nicholas MLC, Agaimy A, Wick MR and Mills SE: Sclerosing microcystic adenocarcinoma of the head and neck mucosa: a neoplasm closely resembling microcystic adnexal carcinoma. Head Neck Pathol 10: 501-508, 2016. PMID: 27233253. DOI: 10.1007/s12105-016-0731-8

2 Schipper JH, Holecek BU and Sievers KW: A tumour derived from Ebner's glands: microcystic adnexal carcinoma of the tongue. J Laryngol Otol 109: 1211-1214, 1995. PMID: 8551161. DOI: $10.1017 /$ S0022215100132475.

3 Hunt J: Pathologic quiz case 1. J Chem Inf Model 53: 1689-1699, 2019. PMID: 25246403. DOI: $10.1017 /$ CBO9781107415324.004

4 Wetter R and Goldstein GD: Microcystic adnexal carcinoma: a diagnostic and therapeutic challenge. Dermatol Ther 21: 452-458, 
2008. PMID: 19076623. DOI: 10.1111/j.1529-8019.2008.00246.x

5 Friedman PM, Friedman RH, Jiang SB, Nouri K, Amonette R and Robins P: Microcystic adnexal carcinoma: collaborative series review and update. J Am Acad Dermatol 41: 225-231, 1999. PMID: 10426893. DOI: 10.1016/s0190-9622(99)70053-8

6 Zhang R, Cagaanan A, Hafez GR and $\mathrm{Hu}$ R: Sclerosing microcystic adenocarcinoma: report of a rare case and review of literature. Head Neck Pathol 13: 215-219, 2019. PMID: 29987694. DOI: 10.1007/s12105-018-0945-Z

7 Weinstein JN, Collisson EA, Mills GB, Shaw KRM, Ozenberger BA, Ellrott K, Sander C and Stuart JM: The cancer genome atlas pan-cancer analysis project. Nat Genet 45: 1113-1120, 2013. PMID: 24071849. DOI: 10.1038/ng.2764

8 Wood A and Conn BI: Sclerosing microcystic adenocarcinoma of the tongue: a report of 2 further cases and review of the literature. Oral Surg Oral Med Oral Pathol Oral Radiol 125: e94e102, 2018. PMID: 29398516. DOI: 10.1016/j.oooo.2017.12.017

9 Chen M Bin and Laber DA: Metastatic microcystic adnexal carcinoma with DNA sequencing results and response to systemic antineoplastic chemotherapy. Anticancer Res 37: 51095111, 2017. PMID: 28870941. DOI: 10.21873/anticanres.11929

10 Chan MP, Plouffe KR, Liu CJ, Palanisamy N, Carskadon S, Zhao L, Nazarian RM, Durham AB, Johnson TM, Andea AA, Patel RM, Lowe L, Fullen DR, Brown NA, Tomlins SA, Udager AM and Harms PW: Next-generation sequencing implicates oncogenic roles for $\mathrm{p} 53$ and JAK/STAT signaling in microcystic adnexal carcinomas. Mod Pathol 30: 1092-1103, 2020. PMID: 31857679. DOI: $10.1038 / \mathrm{s} 41379-019-0424-4$
11 Zhou Y, Shen JK, Hornicek FJ, Kan Q and Duan Z: The emerging roles and therapeutic potential of cyclin dependent kinase 11 (CDK11) in human cancer. Oncotarget 7: 4084640859, 2016. PMID: 27049727. DOI: 10.18632/oncotarget.8519

12 Grulich AE, van Leeuwen MT, Falster MO and Vajdic CM: Incidence of cancers in people with HIV/AIDS compared with immunosuppressed transplant recipients: a meta-analysis. Lancet 370: 59-67, 2007. PMID: 17617273. DOI: 10.1016/S01406736(07)61050-2

13 Chang H, Sasson A, Srinivasan S, Golhar R, Greenawalt DM, Geese WJ, Green G, Zerba K, Kirov S and Szustakowski J: Bioinformatic methods and bridging of assay results for reliable tumor mutational burden assessment in non-small-cell lung cancer. Mol Diagnosis Ther 23: 507-520, 2019. PMID: 31250328. DOI: 10.1007/s40291-019-00408-y

14 Yarchoan M, Albacker LA, Hopkins AC, Montesion M, Murugesan K, Vithayathil TT, Zaidi N, Azad NS, Laheru DA, Frampton GM and Jaffee EM: PD-L1 expression and tumor mutational burden are independent biomarkers in most cancers. JCI Insight 4: 1-10, 2019. PMID: 30895946. DOI: 10.1172/jci.insight.126908

Received August 12, 2020

Revised September 1, 2020 Accepted September 8, 2020 